\title{
Accuracy verification of a simple local three-dimensional displacement measurement method of DIC with two images coordinates
}

\author{
MING-HSIANG SHIH ${ }^{1}$, SHIH-HENG TUNG ${ }^{2, *}$, HAN-WEI HSIAO $^{3}$ and WEN-PEI SUNG ${ }^{4}$ \\ ${ }^{1}$ Department of Civil Engineering, National Chi Nan University, Nan-Tou 545, Taiwan, R.O.C. \\ ${ }^{2}$ Department of Civil and Environmental Engineering, National University of Kaohsiung, Kaohsiung 81148, \\ Taiwan, R.O.C. \\ ${ }^{3}$ Department of Information Management, National University of Kaohsiung, Kaohsiung 81148, Taiwan, R.O.C. \\ ${ }^{4}$ Department of Landscape Architecture, Integrated Research Center for Green Living Technologies, National \\ Chin-Yi University of Technology, Taichung 41170, Taiwan, R.O.C. \\ e-mail: shtung@nuk.edu.tw; drwpsung@yahoo.com.tw
}

MS received 30 June 2015; revised 4 October 2015; accepted 18 November 2015

\begin{abstract}
There are two methods applied for three-dimensional digital image correlation method to measure three-dimensional displacement. One is to measure the spatial coordinates of measuring points by analyzing the images. Then, the displacement vectors of these points can be calculated using the spatial coordinates of these points obtained at different stages. The other is to calibrate the parameters for individual measuring points locally. Then, the local displacements of these points can be measured directly. This study proposes a simple local three-dimensional displacement measurement method. Without any complicated distortion correction processes, this method can be used to measure small displacement in the three-dimensional space through a simple calibration process. A laboratory experiment and field experiment are carried out to prove the accuracy of this proposed method. Laboratory test errors of one-dimensional experiment are similar to the accuracy of the $\mathrm{XYZ}$ table; the error in Z-direction is only $0.0025 \%$ of the object distance. The measurement error of laboratory test is about $0.0033 \%$ of the object distance for local three-dimensional displacement measurement test. Test and analysis results of field test display that in-plane displacement error is only $0.12 \mathrm{~mm}$, and the out-of-plane error is $1.1 \mathrm{~mm}$ for $20 \mathrm{~m} \times 30 \mathrm{~m}$ measuring range. The out-of-plane error is only about $10 \mathrm{PPM}$ of the object distance. These test and analysis results show that this proposed method can achieve very high accuracy under small displacement for both of laboratory and field tests.
\end{abstract}

Keywords. Small displacement; displacement measurement; local calibration; 3D digital image correlation.

\section{Introduction}

In virtue of the raise of the resolution of digital camera and computing speed of computer, there are more and more researchers engaged in the studies of using digital image to measure and analyze displacement and strain variation of test materials and structures recently [1-7].

Georeferencing is the process of associating a physical map or raster image of a map with spatial locations, related to a geographical location, such as points of interest, roads, places, bridges, or buildings [8]. Traditionally, the spatial coordinates of measured points need to be calculated first when three-dimensional digital image measurement method is applied to measure three-dimensional displacement [1, 914]. Then, the displacement vectors of the measured points at various stages can be obtained by subtracting the initial spatial coordinates from the spatial coordinates at each stage.

*For correspondence
This method requires accurate distortion correction and calibration of the camera orientation parameters. This process is complex and has a high technical threshold. Therefore, some of researchers proposed new techniques to improve this complicated procedure of distortion correction and calibrating the orientation parameters of camera: Ortiz et al proposed to apply three-dimensional ray tracing through the different surfaces following denoising segmentation of surface [15], $\mathrm{Xu}$ et al proposed a method, based on the relationship between the coordinates of uniformly scanned points and those of sinusoidally scanned points, for correlating the geometric distortion of images [16]. Zhang \& Shen [17] proposed the changing image coordinates method and object coordinates method, the test results reveal that the changing object coordinates method is less influenced by terrain fluctuation, and its correction accuracy is higher than the changing flight height method and the changing focal length method. Belden [18] proposed a new method for performing bundle adjustment-based calibration of a multi-camera setup 
with refractive interfaces in the optical path. This method is desirable to avoid tedious alignment of calibration grids in multiple locations. Arfaoui and Plante [19] proposed a simple and accurate model based on image coordinate measurement of known three-dimensional position for extracting the optical characteristics and orientation of the camera. Although the above-mentioned methods are accurate for three-dimensional calibration, the mathematical models are complicated. Otherwise, method of traditional georeferencing focuses on local detecting area to process parameter calibration and measurement. Measurement accuracy of this method is not enough to survey the small dispalcement of slope failure. In this research, small absolute displacement vectors are obtained from individual measuring pint to process local calibration and analyze the three-dimensional displacement. This method is not necessary for applying the majority of monitoring or testing techniques to detect the variation of object displacement for obtaining high measurement accuracy of absolute small displacement without any complicated distortion correction process or calibration of inner- and outer-orientation parameters.

Therefore, in order to extend the application of digital image correlation method, a simple local three-dimensional displacement measurement method is proposed in this study. This proposed method only needs to make a local calibration for every individual measured point and measure the local displacement of the measured point directly. Although this method cannot obtain the spatial coordinates of measuring points, it can also be used to measure a small displacement in space after completing a simple calibration process. This local three-dimensional displacement measured method only uses the real displacements and the image coordinates of the measured points on two images to calibrate the necessary parameters. In addition, its application is limited to the measuring of small displacement. Therefore, in case the lens distortion is not very serious, there is no need to carry out any complicated lens distortion correction processes while determining the displacement. In this paper, the mathematical model of this three-dimensional simple local displacement measurement method with two image coordinates is derived, and then the laboratory test is tested and verified to demonstrate the accurate degree of this proposed method. Then, this proposed method is tested and verified by the real field test to demonstrate the accuracy of this proposed method for applying to measure small displacement.

\section{Methodology}

\subsection{Mathematical model of this proposed method}

There are two image coordinates of $P\left(x_{1}^{c}, y_{1}^{c}\right)$ and $Q\left(x_{2}^{c}, y_{2}^{c}\right)$ for two cameras to detect the displacement of object. The plane spot position of test object remains on plane; therefore, the plane equation can be expressed as follows:

$$
Z=a+b X+c Y,
$$

where $X_{j}=\frac{e_{12}}{d_{m, j}} x_{j}, Y_{j}=\frac{e_{12}}{d_{m, j}} y_{j}, Z_{j}=\frac{e_{12}}{d_{m, j}} z_{j}$ and $d_{m, j}$ is moving length of image, $e_{12}$ is translation distance of camera, $\lambda$ is density constant of image sensor. Then, Eq. (1) can be expressed as follows:

$$
\frac{\lambda}{d_{m, j}}=a+b \frac{e_{12}}{d_{m, j}} x_{j}+c \frac{e_{12}}{d_{m, j}} y_{j} .
$$

Appealing for moving length of image $d_{m, j}$ transposes to the left-hand side of equal sign, the other items transpose to the right-hand side of equal sign. Then, the moving length of image can be expressed as follows:

$$
d_{m, j}=A_{j}+B_{j} e_{12} x_{j}+C_{j} e_{12} y_{j}
$$

where $A_{j}, B_{j}$ and $C_{j}$ are unknown constants.

The distance between two cameras, installed at the fixed points to detect the digital images, is constant. Thus, the amount changes of test object between detecting images from the points of these two cameras, $P$ and $Q$, can be obtained by Eqs. (4) and (5) based on the moving length of the test object.

$$
\begin{gathered}
\sum_{j=1}^{2} \Delta x_{j}=x_{j, 2}-x_{j, 1}=\sum_{j=1}^{2} \frac{\operatorname{sign}(\Delta x)}{\sqrt{1+m^{2}}} d_{m, j} \\
\sum_{j=1}^{2} \Delta y_{j}=y_{j, 2}-y_{j, 1}=\sum_{j=1}^{2} \frac{\operatorname{sign}(\Delta x) m}{\sqrt{1+m^{2}}} d_{m, j}
\end{gathered}
$$

where $\operatorname{sign}(\Delta x)$ is plus or minus sign of the amount of image change on $x$ coordinate.

Substituting Eq. (3) into Eqs. (4) and (5), the image coordinates of the object move to the second position which can be expressed as follows:

$$
\begin{aligned}
\sum_{j=1}^{2} x_{j, 2}= & x_{j, 1}+\sum_{j=1}^{2} \frac{\operatorname{sign}\left(\Delta x_{j}\right)}{\sqrt{1+m^{2}}}\left(A_{j}+B_{j} e_{12} x_{j}+C_{j} e_{12} y_{j}\right) \\
\sum_{j=1}^{2} x_{j, 2}= & x_{j, 1}+\left[\frac{\operatorname{sign}\left(\Delta x_{1}\right)}{\sqrt{1+m^{2}}} A_{1}+\frac{\operatorname{sign}\left(\Delta x_{2}\right)}{\sqrt{1+m^{2}}} A_{2}\right] \\
& +\frac{\operatorname{sign}\left(\Delta x_{1}\right)}{\sqrt{1+m^{2}}} B_{1} x_{1}+\frac{\operatorname{sign}\left(\Delta x_{1}\right)}{\sqrt{1+m^{2}}} C_{1} y_{1} \\
& +\frac{\operatorname{sign}\left(\Delta x_{2}\right)}{\sqrt{1+m^{2}}} B_{2} x_{2}+\frac{\operatorname{sign}\left(\Delta x_{2}\right)}{\sqrt{1+m^{2}}} C_{2} y_{2} .
\end{aligned}
$$

Then, simplifying the differentiation on $x$ coordinate, Eq. (7) can be shown as follows:

$$
\mathrm{d} x=a_{0}+a_{1} x_{1}^{c}+a_{2} y_{1}^{c}+a_{3} x_{2}^{c}+a_{4} y_{2}^{c} .
$$

Equivalently, the differentiation on $y$ coordinate can be expressed as follows:

$$
\mathrm{d} y=b_{0}+b_{1} x_{1}^{c}+b_{2} y_{1}^{c}+b_{3} x_{2}^{c}+b_{4} y_{2}^{c} .
$$


Otherwise, according to the assumption of the plane spot position of test object remains on plane, then, the differentiation on $z$ coordinate can be expressed as follows:

$$
\mathrm{d} z=c_{0}+c_{1} x_{1}^{c}+c_{2} y_{1}^{c}+c_{3} x_{2}^{c}+c_{4} y_{2}^{c} .
$$

Actually, the displacement of the measuring point is relatively small when compared with the object distance, the real scale coordinate difference can be assumed to be a linear function of the image coordinates. $a_{0}-c_{4}$ in Eqs. (8)(10) are local three-dimensional transformation parameters. These 15 transformation parameters can be determined through calibration procedures. As long as the cameras are fixed, and the focus and aperture value remain unchanged, the transformation parameters can be continuously used in the following local displacement measurement.

\subsection{Calibration of transformation parameters}

The displacement of the measuring point in the local threedimensional coordinate system can be calculated by using Eqs. (8)-(10). Assume that the measuring point has $N$ controlled displacement vectors within the field of view of both cameras, as shown below:

$$
\left[\mathrm{d} x_{i}, \mathrm{~d} y_{i}, \mathrm{~d} z_{i}\right]^{T}, \quad i=1 \ldots N .
$$

Substituting all image coordinates obtained from these $N$ controlled displacement vectors into Eqs. (8)-(10) leads to $3 N$ independent equations. If $N$ is equal to 5 , a unique solution of these 15 local three-dimensional transformation parameters can be solved. However, there are inevitable image coordinate errors in the DIC measurement process, so we can take more than five controlled displacement vectors to raise the accuracy while solving these 15 transformation parameters. It is suggested that there should be at least three displacements along the local $X-, Y$ and $Z$-direction respectively. Because the equation number is greater than the number of unknown parameters, linear regression analysis can be used to solve the local transformation parameters. Although all measuring points of test object need to be calibrated, measurement accuracy of this proposed method is high and also uncomplicated.

\section{Test and verification}

Traditionally, there are two or more cameras used for threedimensional image measurement methods to capture the images of the observed object, and then the spatial coordinates can be calculated based on the position differences between these images. In this research, the adopted experimental setup is the same as that in the traditional three-dimensional image measurement method. Two cameras are arranged in front of the measuring point, as shown in figure 1. Camera 1 and 2 must be installed on

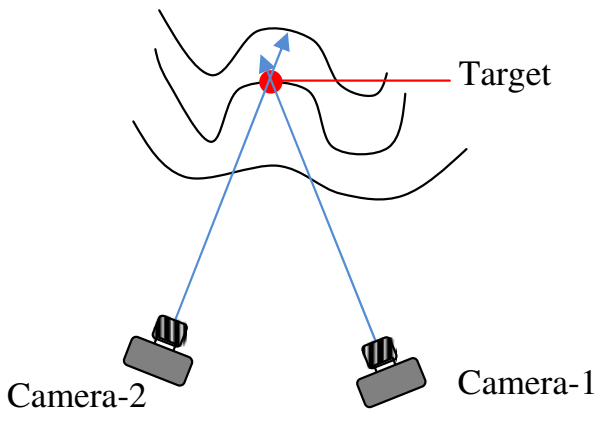

Figure 1. Schematic drawing of instrument arrangement of local three-dimensional image measurement.

stable fixtures or in monitoring stations. In this way, the variation of the relationship between the real scale displacement and the image coordinates according to the movement of camera can be avoided. Thus, the calibration process is carried out for a selected individual point based on the above-mentioned mathematical model. The analysis procedure of this proposed local three-dimensional displacement measurement method is outlined as follows:

(1) Select a local three-dimensional coordinate system with its origin at the measuring point.

(2) Carry out a calibration process to obtain the relationship between the real scale displacement and the image coordinates of the measuring point.

(3) Capture the digital images and calculate the corresponding local three-dimensional displacement.

In order to demonstrate the accuracy of this simplified method for measuring the small displacement in the threedimensional space, the laboratory test is applied to test and verify this proposed method firstly. Then, the confirmatory experiment method of field test is utilized to authenticate the practicability of this proposed method.

\subsection{Laboratory experiment}

3.1a Experimental setup and test procedure: For the laboratory test, a $75 \mathrm{~mm}$ wide and $80 \mathrm{~mm}$ long steel plate with structural speckle is taken as the specimen. The structural speckle with black and white mark is plastered on the surface of the steel plate to detect the small displacement of this test, shown in figure 2. Two cameras, Canon EOS 550D and Canon 7D with Canon EF $50 \mathrm{~mm}$ F 1.8II lens, are applied to detect the digital images of object movement in this test. In order to measure the real displacement of the test object, high-precision XYZ table with precision of $0.01 \mathrm{~mm}$ in all directions and three way camera head are applied in this test. Specimen is mounted on the XYZ table. Two cameras are installed in front of the specimen. The object distance is about $60 \mathrm{~cm}$; the experimental setup is shown in figure 3 . Then, the laboratory test procedure is: (1) select an arbitrary point on the specimen as the observation point; (2) the specimen is moved from $-4 \mathrm{~mm}$ to 


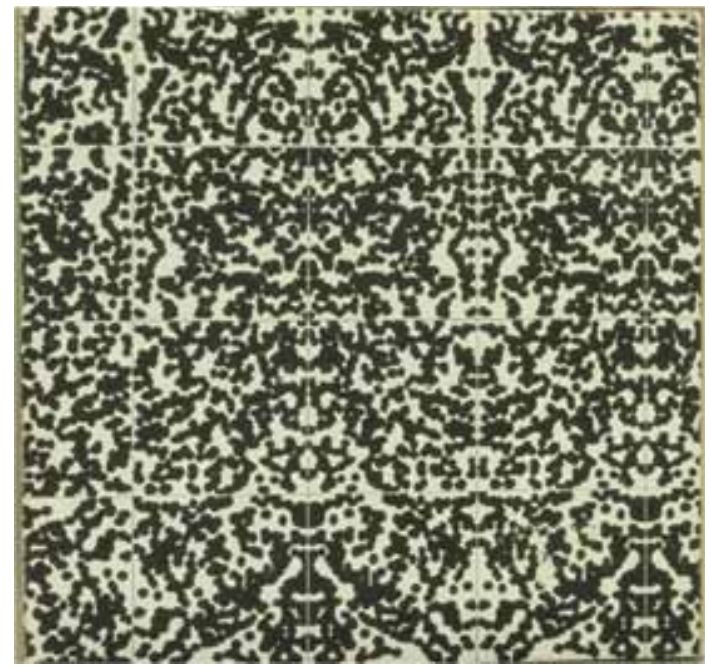

Figure 2. Test specimen.

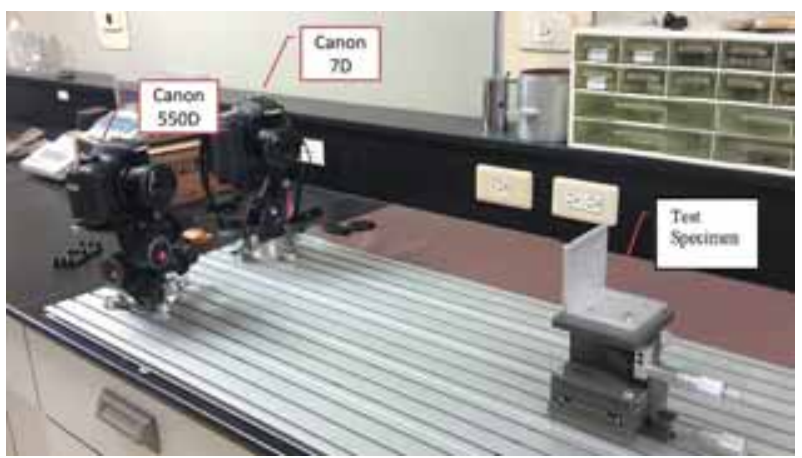

Figure 3. Experimental setup for laboratory test.

$4 \mathrm{~mm}$ in $X$-direction, and the photos are taken at each $0.5 \mathrm{~mm}$ displacement. The same process is then repeated to take pictures in $Y$ - and Z-direction; (3) calculate $a_{0}-a_{4}$, $b_{0}-b_{4}$ and $c_{0}-c_{4}$ by the proposed mathematical model also through linear regression to obtain the values of $a_{0}-c_{4}$; (4) calculate the measurement errors. The image coordinates $\left(x_{1}^{c}, y_{1}^{c}\right)$ and $\left(x_{2}^{c}, y_{2}^{c}\right)$ of the observed point on all photos are substituted into Eqs. (8)-(10). The corresponding estimated displacements can be determined. The differences between the controlled displacements and the estimated displacements are defined as the measurement errors.

\section{1b Experimental results and discussion:}

(1) One-dimensional movement

Typical specimen photo, taken by this experiment, is shown in figure 4 . An arbitrary observation point on the specimen surface is selected. Then, coefficients $a_{0}-c_{4}$, obtained through calibration procedure, are used to calculate the corresponding estimated displacement, and the

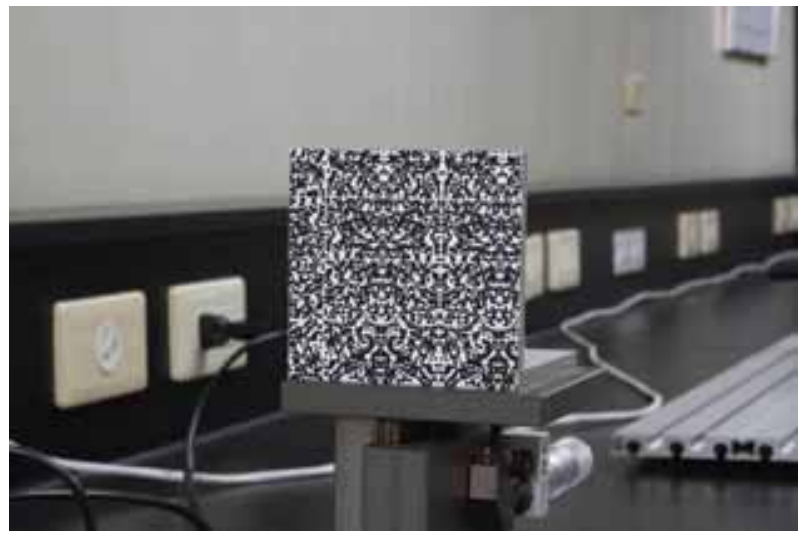

Figure 4. Photo taken in the laboratory experiment.

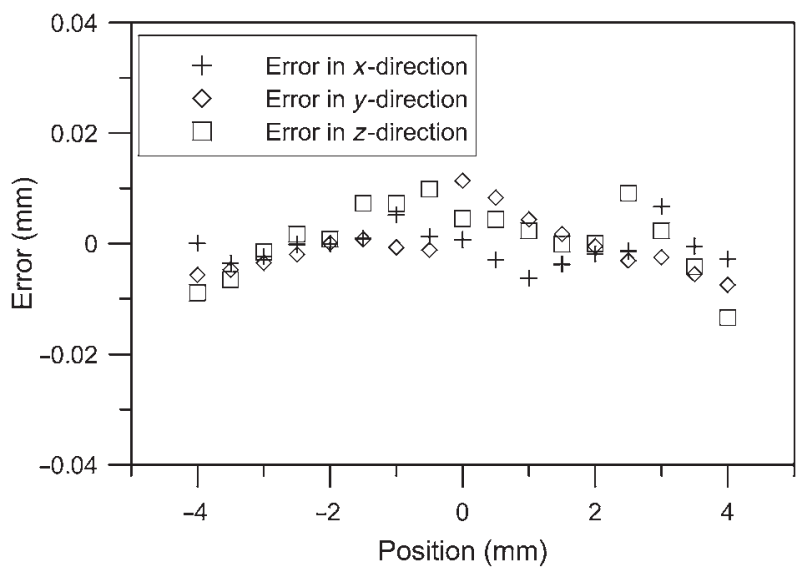

Figure 5. Measurement error while moving in $X$-direction for laboratory test.

analysis results are compared with the actual controlled displacements. The errors at various displacements are shown in figures 5-7. It shows that most of the errors are between $\pm 0.02 \mathrm{~mm}$. The standard deviations of errors in $X$-, $Y$ - and Z-direction are $0.0042,0.0069$ and $0.0075 \mathrm{~mm}$ respectively. The $95 \%$ confidence intervals of the error in $X$-, $Y$ - and $Z$-direction are $\pm 0.0084 \mathrm{~mm}, \pm 0.0137 \mathrm{~mm}$ and $\pm 0.0150 \mathrm{~mm}$ respectively. These errors are similar to the accuracy of the XYZ table. The error in Z-direction is about only $0.0025 \%$ of the object distance. These test results confirm the validity of this proposed method and also display that this proposed method has very high measurement accuracy.

\section{(2) Three-dimensional movement}

Specimen is moved in the three-dimensional space to test and verify the accuracy degree of this proposed method. Digital images are taken at the points as shown in figure 8 . An arbitrary point on the specimen surface is selected for the observation purpose. The transformation parameters, 


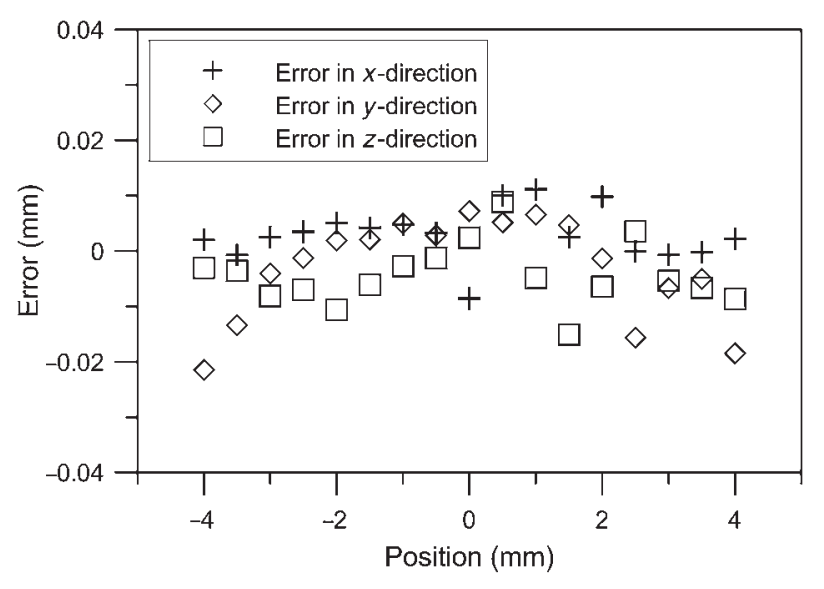

Figure 6. Measurement error while moving in $Y$-direction for laboratory test.

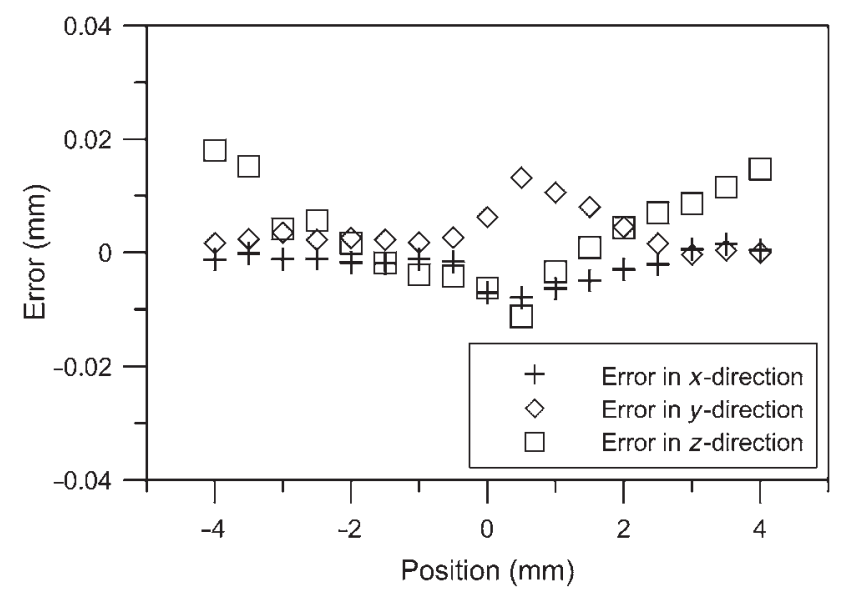

Figure 7. Measurement error while moving in Z-direction for laboratory test.

obtained from Eqs. (8)-(10), are adopted to calculate displacements and errors, and the analysis results are shown in figure 9. It shows that most of the errors in every direction are still within the range of $\pm 0.02 \mathrm{~mm}$, which is about $0.0033 \%$ of the object distance, even when the movement simultaneously occurs in all three directions. The test and analysis results show that the feasibility of this proposed method to the relative three-dimensional displacement measurement of an object has been demonstrated.

From the test results of laboratory experiment, the measurement error is about $0.0025 \%$ of the object distance in Z-direction for one-dimensional test and $0.0033 \%$ of the object distance for local three-dimensional displacement measurement method respectively. These results are obtained from an arbitrarily selected observation point. All test results reveal that this proposed method provides with high accuracy. Therefore, the feasibility of this proposed measurement method can be demonstrated in this laboratory test.

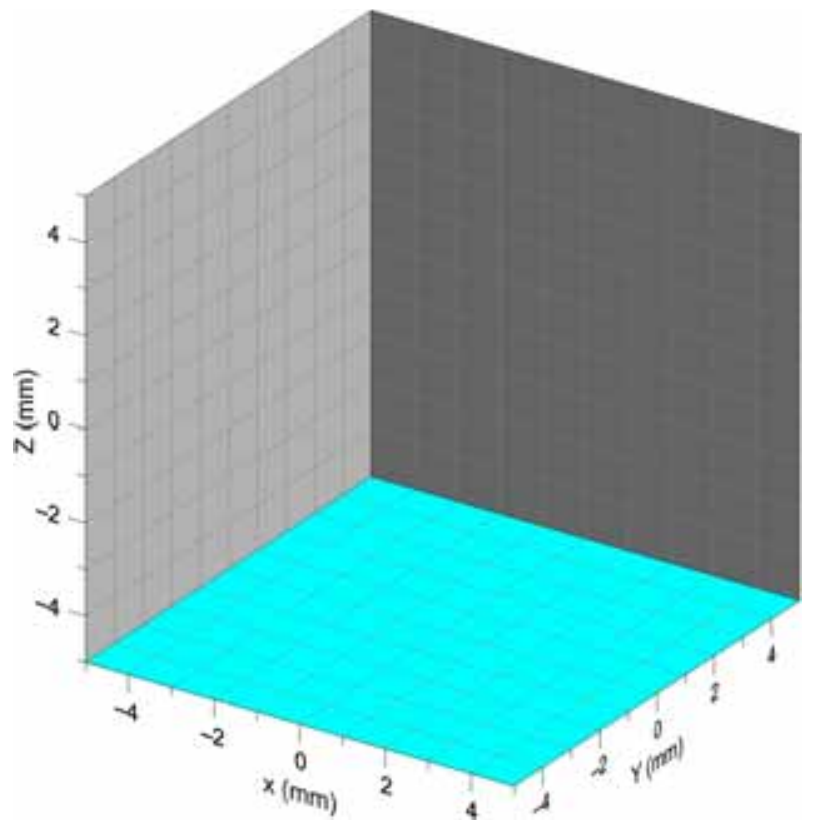

Figure 8. Locations where pictures are taken in three-dimensional movement experiment.

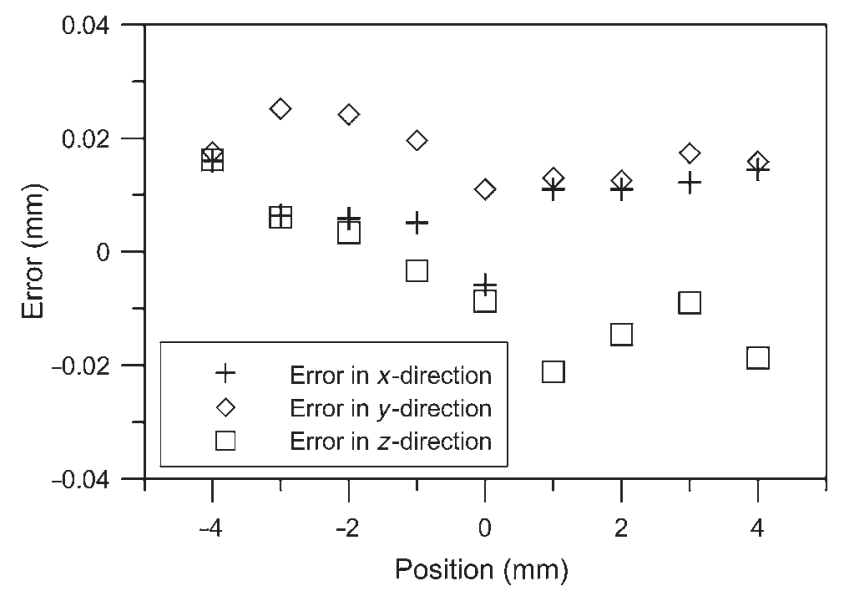

Figure 9. Errors in every direction when movement simultaneously occurs in all three directions.

\subsection{Field experiment}

The digital images, detected by digital cameras, are affected by the air temperature, humidity, change of sunlight and other environmental disturbance. These nature-environment effects are hard to control by human being. Therefore, in order to extend the application of this proposed method for real filed test, field experiment is planned to test and verify the accuracy of this proposed method, applied to measure in situ displacement. The detail setup for this field experiment and test and analysis results are described as below:

3.2a Layout of field experiment: This field experiment is carried out on the hillside area which is outside Earthquake 


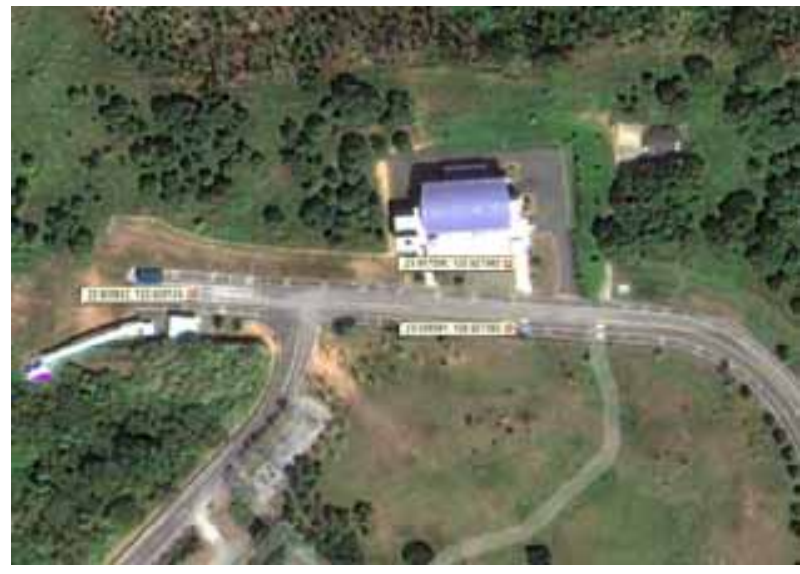

Figure 10. Layout of field test (retrieved from Google Map).

and Disaster Prevention Laboratory of National Chi Nan University in Central Taiwan. The layout of this field experiment is shown in figure 10. The locations of cameras and measuring target and their latitude and longitude coordinates are marked on the map, retrieved from the satellite images of Google Map.

\section{(1) Setup of digital cameras}

There are two Canon EOS 600D cameras with Canon EF $100 \mathrm{~mm} \mathrm{f} / 2.8$ Macro USM lens are adopted as the image acquisition devices, in this study. The object distance (the distance between the middle point of two cameras and the experimental target) is about $115 \mathrm{~m}$, and the distance between two cameras is about $22.7 \mathrm{~m}$, about one-fifth of the object distance. The field of view of the camera is about $30 \mathrm{~m} \times 20 \mathrm{~m}$ while the maximum size of the target is only $80 \mathrm{~cm}$. However, a lens with longer focal length is not used in this test. Because that setup of this field test is much close to that of real situation, when multiple points are observed simultaneously.

\section{(2) Setup of measuring target}

A $60 \mathrm{~cm} \times 80 \mathrm{~cm}$ target plate is installed on a two-way table on the opposite slope of the cameras as the measurement target. The target plate is painted with black and white diamond-shaped blocks with a diagonal length of $5.43 \mathrm{~cm}$ as structural spectacle, as shown in figure 11 . Actually, regular mark is not favorable for digital image correlation (DIC) identification procedure; random mark can help preventing the occurrence of dislocation during identification procedure. Nevertheless, there are some reasons for adopting regular mark in this study. In order to produce a nearly $20 \mathrm{~cm}$ displacement in all three directions, a large XYZ table is needed to be manufactured. However, such a heavy instrument is not suitable for field experiment. Therefore, a two-way table, can move $12 \mathrm{~cm}$ in $X$ direction and $16 \mathrm{~cm}$ in $Z$ direction, is adopted in this research, shown in figure 12. In order to simulate the movement in the

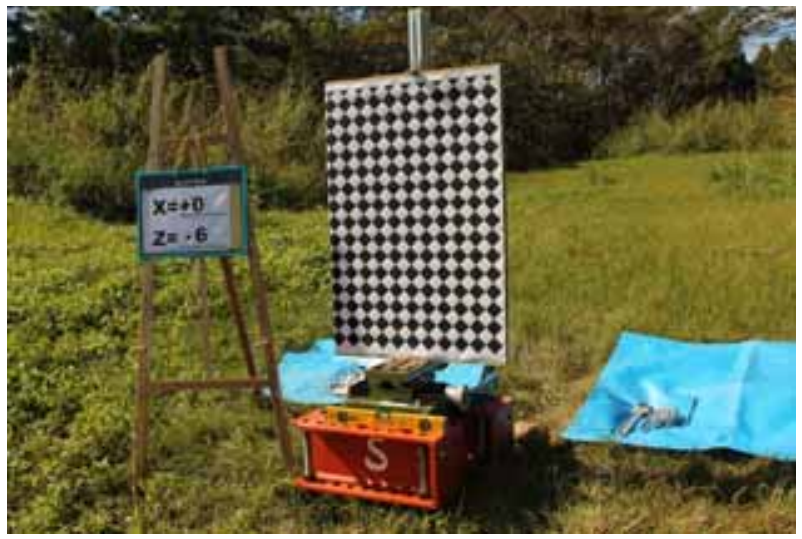

Figure 11. Measurement target and two-way table for field test.

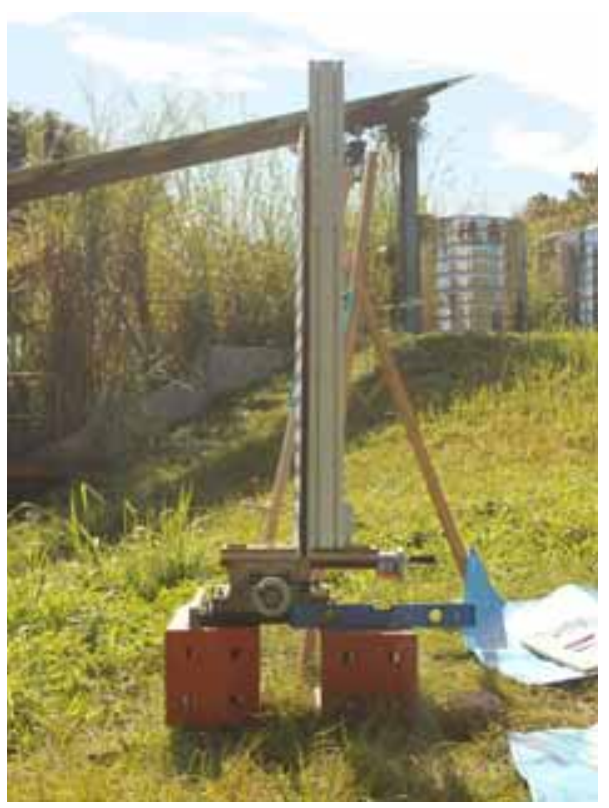

Figure 12. Side view of measuring target and the two-way table for field test.

$Y$ direction, regular and periodic mark is therefore used in this experiment. The wavelength is $5.43 \mathrm{~cm}$, so it can simulate movements in $Y$ direction with a span of $5.43 \mathrm{~cm}$. In order to eliminate the impact of environmental white noise interference, both cameras take 10 photos at each controlled displacement during calibration. The average coordinates obtained from these 10 photos are then used to calculate displacement. The controlled displacements in three directions are shown in table 1 .

3.2b Experimental results: Figure 13 is a typical image, detected from camera to analyze the displacement variation of test object movement. It can be found that the size of the target in this image is very small, only about 120 pixels in width. Therefore, this image is substituted into Eqs. (8), (9) and (10) to obtain the transformation parameters and the 
Table 1. Controlled displacements.

\begin{tabular}{lc}
\hline Direction & Displacement $(\mathrm{cm})$ \\
\hline $\mathrm{X}$ & $-6,-4,-2,0,2,4,6$ \\
$\mathrm{Y}$ & $0,5.43,10.86,16.29$ \\
$\mathrm{Z}$ & $-8-6,-4,-2,0,2,4,6,8$ \\
\hline
\end{tabular}

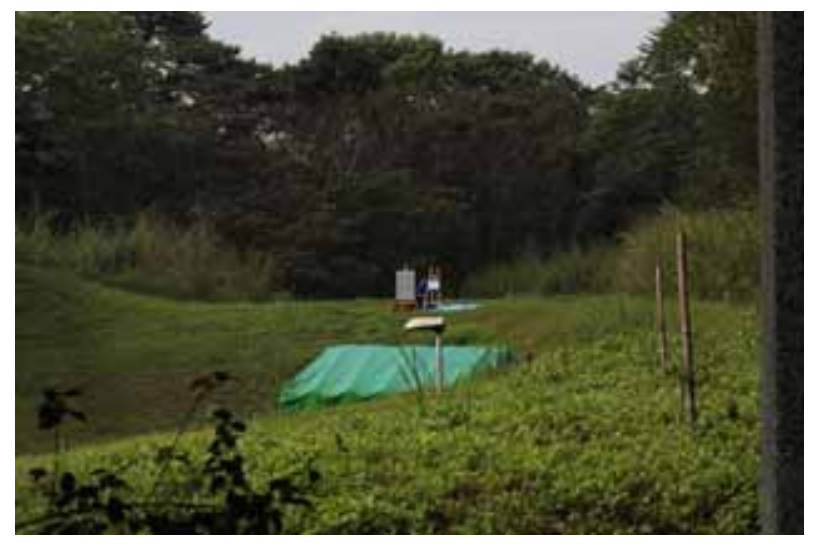

Figure 13. Typical photo for field test.

image coordinates by regression analysis at each controlled displacement respectively. Then, the corresponding displacements and errors can be determined. Table 2 lists the controlled displacement, displacement calculated by linear regression analysis and displacement error.

Table 2 shows that both the maximum displacement errors in $X$-direction and $Y$-direction are about $0.011 \mathrm{~cm}$. The maximum displacement error in Z-direction is $0.11 \mathrm{~cm}$. The standard deviations of the displacement errors in $X-, Y$ -

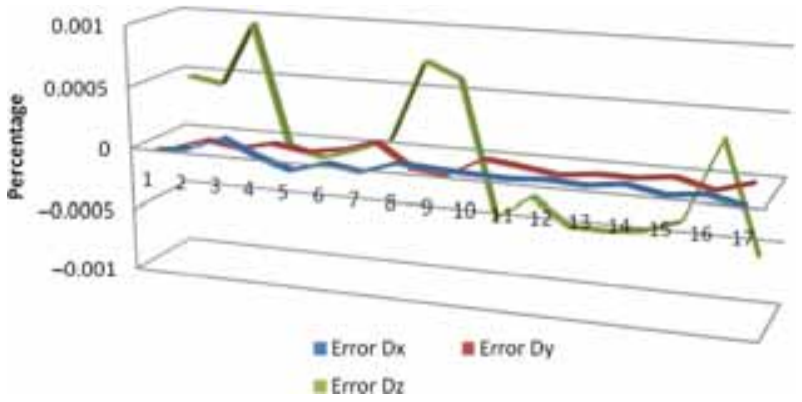

Figure 14. Test errors of field test in three-dimensional analysis.

and Z-direction are $0.005208,0.006368$ and 0.056323 , respectively. The corresponding $95 \%$ confidence interval of displacement errors are 0.010416, 0.012736 and 0.112646 . While the measuring range is about $20 \mathrm{~m} \times 30 \mathrm{~m}$, the inplane displacement error is only $0.12 \mathrm{~mm}$, and the out-ofplane error is $1.1 \mathrm{~mm}$. The out-of-plane error is only about 10 PPM of the object distance. Then, the displacement errors in $X-, Y$ - and $Z$-direction divided by the distance between the middle point of two cameras and the experimental target reveal in figure 14. It shows that the displacement errors relative the object distance is very tiny. It shows that the measurement accuracy of this proposed method of field test is very high. Otherwise, it also displays that the out-of-plane error is about 10 times the values of the other two in-plane errors. This is because the distance between these two cameras is relative small when compared to the distance between camera and target. Hence, the out-of-plane accuracy can be improved by increasing distance between these two cameras.

Table 2. Displacement calculated by linear regression analysis and displacement error (unit: $\mathrm{cm}$ ).

\begin{tabular}{rcccccccc}
\hline DX & DY & DZ & Cal. DX & Cal. DY & Cal. DZ & Error DX & Error DY & Error DZ \\
\hline 0 & 0 & -8 & -0.00343 & -0.00989 & -7.94709 & -0.00343 & -0.00989 & 0.052906 \\
0 & 0 & -6 & -0.00027 & 0.000421 & -5.95265 & -0.00027 & 0.000421 & 0.047348 \\
0 & 0 & -4 & 0.013181 & -0.00448 & -3.89185 & 0.013181 & -0.00448 & 0.108145 \\
0 & 0 & -2 & -0.00102 & 0.002769 & -2.0071 & -0.00102 & 0.002769 & -0.0071 \\
0 & 0 & 0 & -0.01136 & -0.00217 & -0.01616 & -0.01136 & -0.00217 & -0.01616 \\
0 & 0 & 2 & -0.00151 & 0.002861 & 1.993852 & -0.00151 & 0.002861 \\
0 & 0 & 4 & -0.00641 & 0.013056 & 4.006588 & -0.00641 & 0.013056 & 0.00615 \\
0 & 0 & 6 & 0.004646 & -0.00846 & 6.08379 & 0.004646 & -0.00846 & 0.08379 \\
0 & 0 & 8 & 0.002524 & -0.01153 & 8.069395 & 0.002524 & -0.01153 & 0.069395 \\
6 & 0 & 0 & 6.000822 & 0.006169 & -0.05723 & 0.000822 & 0.006169 & -0.05723 \\
4 & 0 & 0 & 3.999818 & 0.002225 & -0.03416 & -0.00018 & 0.002225 & -0.03416 \\
2 & 0 & 0 & 2.002128 & -0.00241 & -0.05759 & 0.002128 & -0.00241 & -0.05759 \\
-2 & 0 & 0 & -1.99976 & 0.001484 & -0.05849 & 0.000237 & 0.001484 & -0.05849 \\
-4 & 0 & 0 & -3.99543 & 0.00135 & -0.0553 & 0.004568 & 0.00135 & -0.0553 \\
-6 & 0 & 0 & -6.00164 & 0.005421 & -0.04251 & -0.00164 & 0.005421 & -0.04251 \\
0 & 10.86 & -0.09 & 0.00232 & 10.8567 & -0.05583 & 0.00232 & -0.0033 \\
0 & 5.43 & -0.09 & -0.0046 & 5.436484 & -0.15767 & -0.0046 & 0.006484 & -0.06767 \\
\hline
\end{tabular}




\section{Conclusions}

The simplified three-dimensional digital image measurement method is proposed in this study. In order to test and verify the accuracy of this proposed mathematical model and test procedure, laboratory test and field test are carried out in this study. There are some conclusions can be synthesized from the above test results:

(1) The results of laboratory experiment show that this method can be used to measure three-dimensional relative displacement. When the specimen is moved in a single direction, the measurement errors in $X, Y$ and $Z$ directions are $0.0084,0.0137$ and $0.015 \mathrm{~mm}$, respectively. When the specimen is moved simultaneously in three directions, almost all of the errors in each direction are less than $0.02 \mathrm{~mm}$, which is approximately $0.0033 \%$ of the object distance. This shows that this proposed method provide with very high measurement accuracy.

(2) When this proposed method is used in field experiment with an object distance of $115 \mathrm{~m}$, this proposed method still has very high measurement accuracy. It shows that this method indeed provide with the practicability for real field monitoring cases.

(3) Although this proposed method is neither suitable for the measurement of absolute coordinates nor for large relative displacement measurement, this method has very high accuracy for small relative displacement measurement and the advantage of lens distortion insensitivity.

From the laboratory and filed test results show that this proposed method provide with very high accuracy for measuring the small relative displacement in one-dimensional movement and three-dimensional movement. The practicality has been proved in this study.

\section{Acknowledgments}

This work is supported under Grant NSC 101-2625-M-260001 and 101-2625-M-167-001 from the Ministry of Science and Technology of Taiwan.

\section{References}

[1] Sutton M A, Orteu J-J and Schreier H W 2009 Image correlation for shape, motion and deformation measurementsbasic concepts, theory and applications. Springer

[2] Sutton M A, Yan J H, Tiwari V, Schreier H W and Orteu J-J 2008 The effect of out of plane motion on 2D and 3D digital image correlation measurements. Opt. Lasers Eng. 46(10): 746-757

[3] Tung S H, Shih M H and Kuo J C 2010 Application of digital image correlation for anisotropic plastic deformation during tension testing. Opt. Lasers Eng. 48: 636-641
[4] Tung S H, Shih M H and Sung W P 2008 Development of digital image correlation method to analyze crack variations of masonry wall. Sadhana-Acad. Proc. Eng. Sci. 33(6): 767-779

[5] Vanlanduit S, Vanherzeele J, Longo R and Guillaume P 2009 A digital image correlation method for fatigue test experiments. Opt. Lasers Eng. 47(3-4): 371-378

[6] Wattrisse B, Chrysochoos A, Muracciole J M and NemozGaillard M 2001 Analysis of strain localization during tensile tests by digital image correlation. Exp Mech. 42(1): 69-80

[7] Weng M C, Tung S H and Shih M H 2009 Microscopic characteristics of problematic Tertiary sandstone as revealed by grain-wide local deformation. Int. J. Rock Mech. 46: 1243-1251

[8] Hackeloeer A, Klasing K, Krisp JM and Meng L 2014 Georeferencing: a review of methods and applications. Ann. GIS 20(1): 61-69. doi:10.1080/19475683.2013.868826

[9] Kahn-Jetter Z L and Chu T C 1990 Three-dimensional displacement measurements using digital image correlation and photogrammic analysis. Exp. Mech. 30(1): 10-16

[10] Luo P F, Chao Y J, Sutton M A and Peters W H 1993 Accurate measurement of three-dimensional deformations in deformable and rigid bodies using computer vision. Exp. Mech. 33(2): 123-132

[11] Orteu J-J 2009 3-D computer vision in experimental mechanics. Opt. Lasers Eng. 47(3-4): 282-291

[12] Tay C J, Quan C, Huang Y H and Fu Y 2005 Digital image correlation for whole field out-of-plane displacement measurement using a single camera. Opt. Commun. 251(1-3): 23-36

[13] Tung S H and Shih M H 2011 Precision verification of a simplified three-dimensional DIC method. Opt. Lasers Eng. 49(7): 937-945

[14] Winter D 1993 Optische Verschiebungsmessung nach dem Objektrasterprinzip mit Hilfe eines fächenorientierten Ansatzes. PhD thesis, Technische Universität Braunschweig, Germany

[15] Ortiz S, Siedlecki D, Grulkowski I, Remon L, Pascual D, Wojkowski M and Marcos S 2010 Optical distortion correction in Optical Coherence Tomography for quantitative ocular anterior segment by three-dimensional imaging. Opt. Express 18(3): 2782-2796

[16] Xu L, Tian X, Li X, Shang G and Yao J 2011 Geometric distortion correction for sinusoidally scanned images. Measur. Sci. Technol. 22(11). doi:10.1088/0957-0233/22/11/ 114023

[17] Zhang Y and Shen X 2013 Approximate correction of length distortion for direct georeferencing in map projection frame. IEEE Geosci. Remote Sens. Lett. 10(6): 1419-1423

[18] Belden J 2013 Calibration of multi-camera systems with refractive interfaces. Exp. Fluids 54: 1463. doi:10.1007/ s00348-013-1463-0

[19] Arfaoui A and Plante F 2011 Camera calibration using composed cubic splines. Geomatica 65(2): 189-197 